\title{
50 ans de la Société Suisse d'Anesthésiologie et de Réanimation (SSAR)
}

\author{
T. Pasch, F. Frei
}

\section{L'anesthésie dès 1945}

Peu après la seconde guerre mondiale, le développement de l'anesthésie dans la plupart des pays européens continentaux accuse un grand retard par rapport aux pays anglo-américains et scandinaves. Si la médecine helvétique de la préguerre est fortement influencée par ses voisins tels la France, l'Allemagne et l'Autriche, les progrès anesthésiques souhaités se doivent toutefois d'être recherchés auprès des régions linguistiques anglo-saxonnes. Durant la guerre, les échanges internationaux se sont avérés difficiles voire impossibles; de plus, la pratique même de l'anesthésie est dévolue aux chirurgiens qui délèguent fréquemment cette tâche à leurs infirmières de narcose. Il incombe occasionnellement aux assistants de procéder à l'anesthésie; toutefois, peu d'entre eux n'y montrent d'intérêt, dans la mesure où la narcose n'est pas considérée comme un acte médical. De ce fait l'anesthésiologie ne progresse guère, l'anesthésie locale et loco-régionale domine et les indications opératoires se doivent d'être restreintes et bien pensées.

Bien que l'administration de curare et l'intubation endotrachéale fasse déjà partie intégrante de l'anesthésie générale moderne en Angleterre, en Amérique et au Canada, ce savoir, quoique porté en haute estime, ne fit son entrée en Suisse que de manière hésitante et ponctuelle. L'Académie Suisse des Sciences Médicales (ASSM), sous la présidence du Professeur Gigon (Bâle), ainsi que quelques rares chirurgiens universitaires ou praticiens privés prennent conscience dans les années 40 déjà des besoins importants d'une évolution dans le domaine de l'anesthésie. Raison pour laquelle l'ASSM décida en 1946 d'envoyer un boursier suisse pour une période prolongée à Boston chez H. K. Beecher. W. Hügin ayant montré son intérêt pour les curares lors de son assistanat en chirurgie, bénéficia de cette bourse et fut nommé en 1949, après un court séjour en Suède et en Angleterre, directeur de la division d'anesthésie de l'hôpital chirurgical universitaire de Bâle. Quelques jeunes collègues se formèrent à leurs propres initiatives, tels Ch. Bovay aux Etats Unis et K. Zeller en Angleterre. Le premier s'installa en pratique privée à
Lausanne, alors que Zeller en 1949 occupa le poste nouvellement créé de Médecin associé en anesthésie à Winterthur. K. Zimmermann apprit les techniques anesthésiques modernes à l'hôpital de la Croix Rouge de Zürich grâce à un anesthésiste anglais invité pour une année; il compléta sa formation dans ce domaine grâce à une collaboration avec deux collègues anglais, puis directement en Angleterre. Par la suite, il dirigea l'anesthésie entre 1951 et 1975 à l'hôpital de la Croix Rouge de Zürich, avant d'occuper de 76 à 87 la présidence du Comité Central de la Fédération des Médecins Suisses. F. Kern se forma entre 1951/52 à Copenhague et Oxford puis reprit le service d'anesthésie de l'hôpital cantonal de Saint-Gall, où il devint en 1959 médecin chef et premier directeur d'un service d'anesthésie indépendant en Suisse. Berne resta un terrain rocailleux durant de nombreuses années aussi bien en milieu universitaire que privé. Il en sera de même de l'hôpital universitaire de Lausanne. L'hôpital cantonal de Genève engagea en 1949 un collègue anglais pour la clinique chirurgicale; il y restera un an. A l'hôpital de Zürich, le développement de l'anesthésie moderne évolua par étapes. Le neurochirurgien H. Krayenbühl a pratiqué des narcoses sous intubation dès 1937; il avait acquis à Londres cette méthode en complément de ses techniques opératoires. K. Mülly, lors d'un semestre de formation en chirurgie thoracique à Stockholm, apprit les techniques anesthésiques utilisées lors de chirurgie thoracique et abdominale haute et les appliqua pour la première fois en chirurgie en automne 1947. Il resta pourtant chirurgien thoracique et se retira de l'anesthésie à l'arrivée de G. Hossli en 1951/52.

\section{La fondation de la société de spécialité}

Les rares anesthésistes de cette période furent considérés de manière extrêmement variable par leurs collègues chirurgiens. Certains étaient convaincus de la qualité des narcoses modernes, mais la plupart des chirurgiens n'avaient guère de compréhension envers cette évolution. Se contentant de ce qui existait, on craignait pour les structures établies en salle d'opération comme en clinique, et plus encore on redoutait des pro- 
Figure 1

Dr Charles Bovay (né en 1920), premier président de la SSAR.
Figure 2

Dr Karl Zimmermann (1922-1999), premier secrétaire de la SSAR et entre 1976 et 1987 président de la FMH

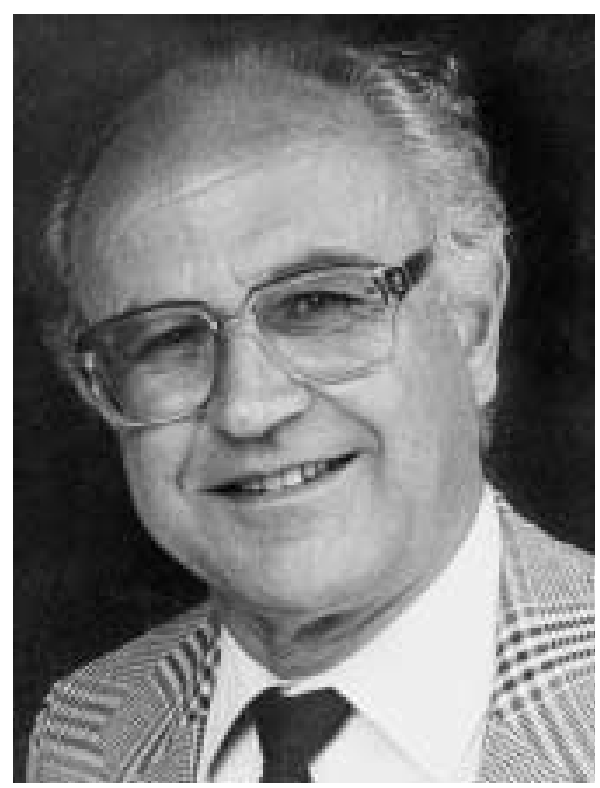

Figure 3

Professeur Werner Hügin (1918-2001), premier caissier de la SSAR.

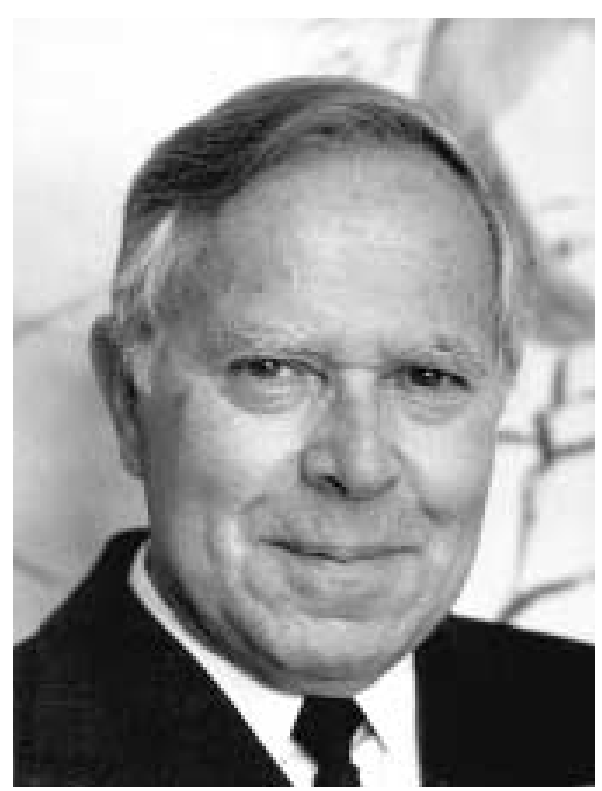

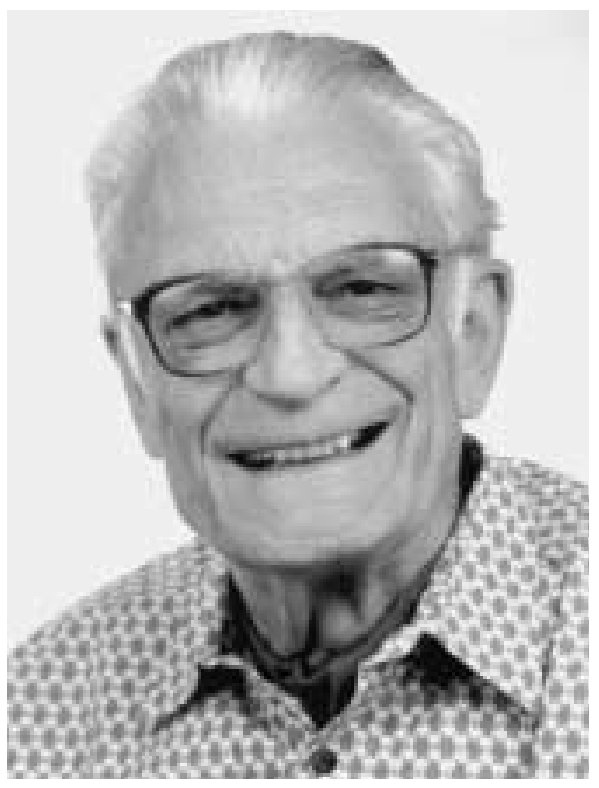

blèmes financiers. Tout cela instigua la petite volée d'anesthésistes à se réunir dans le dessein d'une part de faire connaissance et d'autre part de défendre ensemble les intérêts légitimes de cette nouvelle spécialité. Le 11 février 1951, Bovay, Hügin et Zeller à Berne décident de fonder un Groupement Professionnel des Anesthésiologues Suisses (GPAS). Hügin fut chargé d'en élaborer les statuts; ceux-ci furent agréés le 15 mars 1951 et entrèrent en vigueur immédiatement. A l'occasion des journées annuelles de la Société Suisse de Chirurgie des 6/7 juillet 1951, se tint la première réunion générale du GPAS à Frauenfeld, durant laquelle plusieurs membres ordinaires et extraordinaires furent admis. Parmi les premiers furent P. König (Genève) et K. Zimmermann.

Il apparut déjà à Frauenfeld que la durée de vie du GPAS serait brève dans la mesure où la Société Suisse de Chirurgie à la même période décida de fonder une section pour l'anesthésie chargée de s'occuper principalement des problèmes scientifiques de l'anesthésie, tandis que les questions professionnelles du GPAS pouvaient ainsi être ignorées. Un esprit de contestation s'éleva dès lors à l'encontre de l'intention manifeste de domination de la part des chirurgiens. C'est pourquoi Hügin entreprit par précaution au début 1952 déjà d'élaborer une proposition de statuts comprenant des critères de formation post-graduée en vue de la fondation d'une Société Suisse d'Anesthésiologie. La fondation d'une Société Autrichienne d'Anesthésie en réponse aux réticences des chirurgiens le 19 octobre 1951 donna en élan supplémentaire à la tentative de la commission professionnelle de se convertir en Société.

En juillet 1952 eut lieu la 39e réunion annuelle de la Société Suisse de Chirurgie à laquelle le GPAS fut convié. A l'invitation étaient joints les statuts d'une section pour l'anesthésie à laquelle les anesthésistes ne pourraient participer qu'en tant que membres associés de la Société Suisse de Chirurgie. Dès lors la création définitive de la Société Suisse d'Anesthésiologie fut décidée, et ce un peu plus d'une année seulement après la naissance du GPAS. La présidence de la réunion de fondation du 5 juillet 1952 fut assurée par le secrétaire du GPAS, K. Zeller. Les membres ordinaires présents votèrent la décision de fondation et la dissolution du GPAS simultanément. Ch. Bovay fut nommé premier président (fig. 1), K. Zimmermann secrétaire (fig. 2) et W. Hügin caissier. Les statuts soumis furent acceptés au prix de légères modifications et définitivement mis en vigueur suite à un référendum en septembre 1952. La société comprend dès lors 7 membres ordinaires, 9 membres extraordinaires et deux membres associés. 
Tableau 1

L'occupation des chaires d'anesthésiologie dans les différentes facultés.

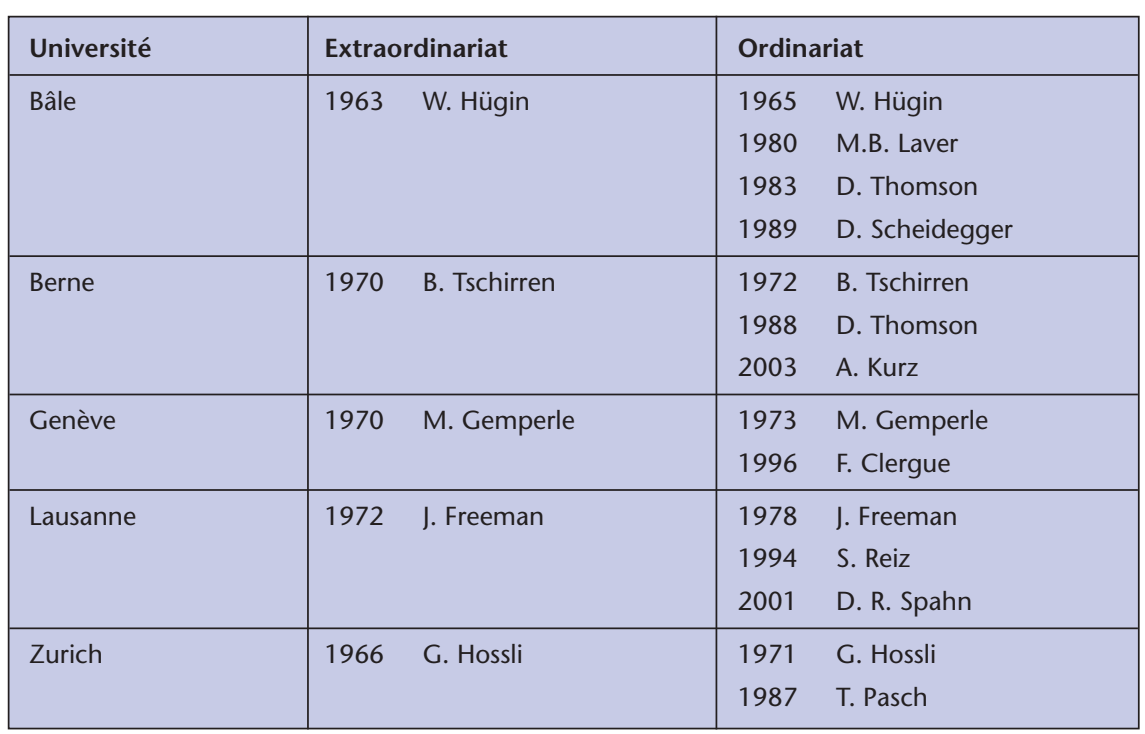

\section{Premiers stades de l'évolution}

L'un des premiers devoirs de la nouvelle société de spécialité fut d'établir des conditions de reconnaissance de l'anesthésie en tant que spécialité. Elle obtint que le premier programme de formation post-graduée officiel pour le titre de spécialiste FMH entre en vigueur le $1^{\text {er }}$ janvier 1954 avec une clause d'état de transition jusque-là. Quatre ans de formation post-graduée dont deux ans en anesthésiologie et une obligation de séjour à l'étranger d'une durée minimale de 6 mois étaient requis; cette dernière clause ne fut supprimée qu'en 1962. En plus des critères de formation, de nombreuses questions de politique professionnelle devaient occuper la société durant les quelques dizaines d'années suivantes; parmi celles-ci, les relations avec les chirurgiens et autres spécialités opératoires, la position des anesthésistes dans la hiérarchie hospitalière, les conditions d'engagement et de contrat, les honoraires et les questions tarifaires, la collaboration avec le personnel infirmier anesthésiste, l'extension du domaine de la spécialité à la médecine d'urgence, à la médecine intensive et à la clinique de la douleur et finalement le développement de la recherche et de l'enseignement. Dans tous ces domaines, un travail de pionnier devait être effectué; bien que les progrès furent marquants, certains échecs ne purent être évités. Un concept de l'anesthésiologie s'impose de plus en plus en tant que description de la spécialité: l'anesthésiologie ne se limite pas à la narcose (anesthésie au sens restreint du terme) mais s'étend éga- lement à la médecine d'urgence et intensive et plus tard à la clinique de la douleur, sans besoin d'exclusivité dans ces domaines. En 1967 on élargit le nom de la société à celui de Société Suisse d'Anesthésiologie et de Réanimation (SSAR). Dans les années 60 , on constate une indépendantisation organisationnelle progressive et inéluctable de la chirurgie, quoique d'intensité variable selon les endroits. En 1967, la SSAR édite des «Directives contractuelles entre anesthésistes et hôpitaux» dans lesquelles des égalités de contrat entre les anesthésistes et les médecins chefs d'autres disciplines de l'hôpital sont exigées. Ce but ne fut que progressivement atteint durant les 35 années suivantes et de manière non uniforme dans l'ensemble du territoire; dans plusieurs endroits, les résistances de la direction médicale et des médecins chefs chirurgicaux ne purent en effet être vaincues.

\section{Relation universitaire}

Il faudra 10 à 15 ans pour que l'anesthésiologie soit totalement admise en tant que discipline académique dans le monde universitaire. En 1957, W. Hügin à Bâle et M. Junod à Genève et en 1960 G. Hossli furent habilités. Une chaire d'enseignement à la Faculté de Médecine Suisse fut octroyée quelques années plus tard. En 1963, Hügin fut nommé professeur extraordinaire à la Faculté de Médecine et directeur de l'institut d'anesthésiologie de l'Hôpital Cantonal de Bâle; deux ans plus tard il fut promu professeur ordinaire. Les différents occupants de la chaire d'enseignement d'anesthésiologie dans les cinq facultés de médecine des universités Suisses sont présentés dans la table 1 . On dénote précocement un effort commun, dans les contrées germanophones, pour être reconnu sur le plan scientifique au niveau local, national et international. En 1952, R. Frey (Mainz), W. Hügin et O. Mayrhofer (Vienne) fondèrent le journal «Der Anaesthesist»; dans un premier temps, organisme autrichien, il fera partie intégrante une année plus tard des sociétés de spécialité allemande et helvétique. Les anesthésistes de la Romandie créèrent au travers d'une édition spéciale annuelle du journal «Médecine et Hygiène» leur propre forum de publications.

Il fut décidé en 1953 d'établir désormais une journée commune biennale des sociétés d'anesthésie autrichienne, allemande et suisse qui devint traditionnelle jusqu'en 1995 où elle prit fin devant la «globalisation» de la science. 


\section{Stades suivants de l'évolution}

Durant les 50 années d'existence de la SSAR, les tâches de la société ne se sont pas uniquement limitées au constant développement et à l'approfondissement de la spécialité médicale, mais se sont également étendues au développement de relations politiques professionnelles. Il n'est pas rare en ce domaine que la SSAR ne tende à anticiper sur ce que les autres spécialités médicales et la FMH n'effectueront que plus tard. De nombreux aspects dans les domaines de la formation, de la sécurité et de l'assurance de qualité sont exemplaires. En 1977 déjà fut décidée l'introduction d'un examen de spécialité qui eut lieu pour la première fois en 1979/80. En 1984, la réunion générale de la SSAR décida de requérir auprès de la FMH l'établissement d'un examen de spécialité obligatoire et l'édition d'un nouveau règlement d'examen. En 1986, la FMH accéda à cette requête; il existe dès lors en anesthésie, et ce parmi les premiers domaines de spécialité en Suisse, un examen oral et écrit obligatoire. Dans le dessein d'assurer et d'améliorer la qualité, la SSAR décida en 1988 d'ajouter l'examen européen à la partie écrite de l'examen suisse. Cet examen existait depuis 1984 au sein de l'Académie Européenne d'Anesthésiologie; sa réussite est requise pour l'obtention du Diplôme Européen en Anesthésiologie et Réanimation. L'Académie fut fondée en 1978 en tant qu'organisation des anesthésistes académiques d'Europe. Par ailleurs, ce n'est que le $1^{\text {er }}$ janvier 1993 que la FMH accepta de sanctionner ce nouveau programme de formation post-graduée permettant d'accéder au titre FMH de médecin spécialiste en anesthésiologie. Celle-ci imposa dès 1995 à toutes les spécialités médicales l'instauration d'un examen de spécialité, soit 9 ans après l'anesthésiologie. Depuis 2000, et ce, tous les 7 ans ou lors du changement de médecin chef ou de catégorie d'hôpital de formation, la commission de la SSAR impose une équipe visiteuse chargée d'examiner les différents établissements en vue d'une reconnaissance en tant que lieu de formation. Cette initiative également sera retenue par la FMH qui éditera sa propre ordonnance de formation le $1^{\text {er }}$ janvier 2002

En 1986, la présidence de la SSAR adopte des «recommandations sur les besoins en personnel en anesthésie dans les hôpitaux ouverts» ainsi que des «directives concernant le monitorage minimal peropératoire», deux mois avant les «Standards for basic intraoperative monitoring» de l'American Society of Anesthesiologists qui prendront bientôt un caractère d'exemple dans le monde entier. Après de nombreuses discus- sions furent adoptés en 1993 des «standards et recommandations» traitant des questions importantes de qualité structurelle et de procédures, tels que par exemple la structure du personnel, les exigences requises pour le personnel et les standards minimaux. Ces nouvelles directives, grâce à leur généralisation et leur développement logique, contribuèrent de manière importante à l'accumulation de controverses et de malentendus entre les deux groupes professionnels médecin et infirmiers anesthésistes. Entre autres, les principes adoptés en 1990 des «limitations de compétence entre médecins et personnel infirmier anesthésistes», donnèrent lieu à de vives oppositions des deux parties, bien que leur contenu soit en harmonie avec le "profil professionnel infirmière anesthésiste infirmier anesthésiste» de l'Association Suisse des Infirmières et Infirmiers agréée en 1989 par la Présidence Centrale de cette association et la SSAR. En novembre 2002 une version retravaillée et élargie de ces standards de même que des recommandations en ce qui concerne le «monitorage et les soins post-anesthésies» a été éditée.

Dès 1995, les exigences de qualité et de sécurité en anesthésie furent traitées avec persévérance au travers de divers projets. On fonda une commission responsable du maintien des exigences de qualité et de la sécurité en anesthésie. Celle-ci développa un système suisse de collection de données au moyen d'un «minimal data set» et d'un système de rapport d'incidents critiques en anesthésie. Ce système put être inclus sur la Website de la SSAR en tant que «Critical Incident Reporting System»(CIRS). Cette idée a également été adoptée par la FMH qui souhaite créer sa propre banque de données de CIRS. Un pas supplémentaire sera la création en 1999 d'une commission analysant les cas de responsabilité civile. Cette commission collecte et juge selon un schéma fixe les cas résolus de responsabilité civile et transmet ces informations aux anesthésistes praticiens dans l'idée de contribuer à l'amélioration de la sécurité des patients. Grâce à l'engagement du président de cette commission H. Schaer, les plus grands assureurs de responsabilité civile sont désormais ouverts à une collaboration. Finalement et à l'initiative de B. Meister fut créée en janvier 2001 une «Fondation pour la sécurité des patients en anesthésie» à la présidence de laquelle il fut lui-même élu. Le soutien d'activités vouées à l'amélioration de la sécurité de l'anesthésie pour les patients dépend de donations. C'est pourquoi l'existence de telles activités se doit d'être dévoilée librement à tous les cercles intéressés. En tant que soutien de la 
fondation pourraient être sollicitées hormis la SSAR, l'Organisation Suisse des Patients, l'Association Suisse des assurances ainsi que les firmes pharmaceutiques et de techniques médicales. Grâce aux médias, le lancement de cette fondation a reçu un écho réjouissant; elle a été reconnue à caractère d'exemple.

\section{Présent et futur}

Nous nous devons à l'heure actuelle d'attester le courage et la vision d'avenir des 14 médecins fondateurs en 1952 de la Société Suisse d'Anesthésiologie. Ils ont su initier un développement qu'ils souhaitaient mais auquel ils n'auraient pu s'attendre à l'époque. A partir d'un germe inapparent s'est développé durant les 50 dernières années un arbre plein de vitalité, doté d'un tronc solide et de nombreuses branches, fleurs et fruits. De la Société Suisse d'Anesthésiologie de 1952 est née la Société Suisse d'Anesthésiologie et de Réanimation de 2002 comprenant plus de 700 membres. En 50 ans, elle est devenue 50 fois plus grande, comme le montre le tableau $\mathrm{n}^{\circ} 2$.

En 2002, l'anesthésiologie suisse se présente comme une spécialité indépendante, confiante et attractive de la médecine. Elle n'est pas limitée à la pratique d'anesthésies au sens étroit du terme, mais au contraire participe considérablement au maintien pré- et postopératoire des patients, l'image professionnelle a progressivement évolué en direction d'une médecine périopératoire. La médecine d'urgence et la médecine intensive, de même que le traitement aigu et chronique de la douleur, sont des composantes établies de la spécialité, sans qu'une quelconque exclusivité ne soit exigée dans ces domaines. Les anesthésistes ont acquis au sein de la «Communauté médicale» une reconnaissance certaine et ont gagné du poids. Autrefois pratiquement quotidiennes, les controverses menaçantes au sein des territoires chirurgicaux et en particulier avec leurs représentants sont devenues plus rares et plus neutres. Une estime associée à une reconnaissance réciproque des compétences et des responsabilités du partenaire sont devenues la règle plutôt que l'exception. La réputation scientifique est de bon niveau, non seulement sur un plan national mais également international. De même l'efficacité des standards de sécurités et la formation post-graduée et continue se distinguent par leur qualité supérieure.

De plus en plus, une évolution rapide devient un facteur déterminant de notre pratique. Cela concerne les procédures cliniques et les bases scientifiques de notre spécialité, ainsi que celles des spécialités voisines et de nos partenaires chi-
Table 2

Progression par décade du nombre de membres (toutes catégories) de la SSAR.

\begin{tabular}{|l|l|}
\hline Année & Nombre de membres \\
\hline 1952 & 14 \\
1962 & 77 \\
1972 & 154 \\
1982 & 296 \\
1992 & 478 \\
2002 & 715 \\
\hline
\end{tabular}

rurgicaux, voire finalement celles de la médecine dans sa globalité. Les progrès de la médecine ne sont que partiellement responsables de la rapidité des changements. La structure globale de la santé publique est bien davantage touchée par des bouleversements constants sans direction définie, actionnés par diverses forces sociales, scientifiques et politiques. Progressivement, ce ne sera plus seulement perçu comme une intrusion par les seuls anesthésistes qui se voient fréquemment livrés à leur destin. Certains problèmes actuels et urgents se doivent d'être cités: les modifications du système tarifaire et de remboursement; la discrépance croissante entre le budget hospitalier ou du service et les exigences constamment croissantes des performances; les réglementations du temps de travail et leurs répercussions sur la qualité de travail et de formation; d'un côté les difficultés de recrutement et de l'autre les craintes d'un excès de médecins spécialistes disponibles sur le marché. Toutefois, on pronostique un manque menaçant d'anesthésistes dans de nombreux pays européens.

La SSAR doit et veut relever ce défi, par lequel elle maintiendra et renforcera ses forces préexistantes comme la reconnaissance précoce des évolutions et leur aménagement, la définition à long terme de buts et leurs modifications. La fondation sus-citée pour la sécurité des patients en anesthésie est un exemple de parade de projet futuriste, dont le caractère de pionnier est à cela reconnaissable que la $\mathrm{FMH}$, en association avec les autorités fédérales veut bientôt inaugurer un projet semblable à un niveau national. Le 14 novembre 2002, lors de son congrès annuel à Lausanne, la SSAR a fêté ses 50 ans d'existence et a publié à cette occasion un document dont les auteurs non seulement lancent un regard retrospectif sur le développement historique mais également prospectif sur les prochaines prises de position de la spécialité et de la Société de spécialité pour l'année 2002 [1].

Nous adressons nos sincères remerciements à la Dresse. Dominique Bettex pour la traduction en français. 